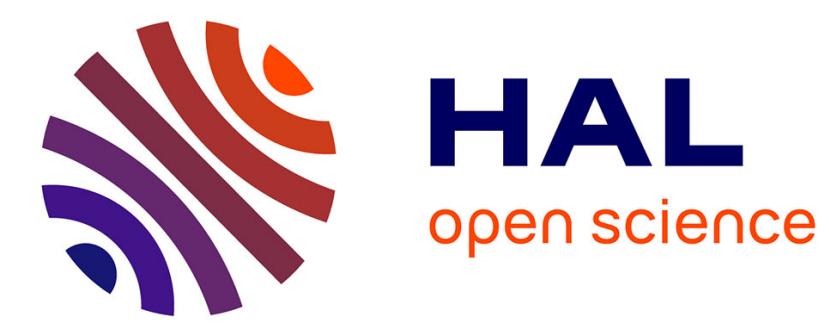

\title{
Online Learning for QoE-based Video Streaming to Mobile Receivers
}

Nesrine Changuel, Bessem Sayadi, Michel Kieffer

\section{To cite this version:}

Nesrine Changuel, Bessem Sayadi, Michel Kieffer. Online Learning for QoE-based Video Streaming to Mobile Receivers. GLOBECOM 2012, Dec 2012, Anaheim, United States. pp.1-5. hal-00727798

\section{HAL Id: hal-00727798 \\ https://hal-centralesupelec.archives-ouvertes.fr/hal-00727798}

Submitted on 4 Sep 2012

HAL is a multi-disciplinary open access archive for the deposit and dissemination of scientific research documents, whether they are published or not. The documents may come from teaching and research institutions in France or abroad, or from public or private research centers.
L'archive ouverte pluridisciplinaire HAL, est destinée au dépôt et à la diffusion de documents scientifiques de niveau recherche, publiés ou non, émanant des établissements d'enseignement et de recherche français ou étrangers, des laboratoires publics ou privés. 


\section{Online Learning for QoE-based Video Streaming to Mobile Receivers}

\author{
Nesrine Changuel, Bessem Sayadi \\ Alcatel-Lucent - Bell-Labs France, \\ Route de Villejust, F-91620 Nozay
}

\author{
Michel Kieffer \\ L2S, CNRS - SUPELEC - Univ Paris-Sud, F-91192 Gif-sur-Yvette \\ LTCI, CNRS - Télécom ParisTech, F-75013 Paris
}

\begin{abstract}
This paper proposes a cross-layer control mechanism to stream efficiently scalable videos to mobile receivers. Its goal is to maximize the quality of the received video while accounting for the variations of the characteristics of the transmitted content and of the channel. The control problem is cast in the framework of Markov Decision Processes. The optimal actions to apply to the system are learned using reinforcement learning. For that purpose, the quality of the decoded frames at receiver is inferred by an observation (i) of the quality of the various scalability layers and (ii) of the level of queues at the Application and Medium Access Control layers of the transmitter only. Delayed as well as absence of information on the channel state are considered. Experiments show that the performance of the proposed solution is only slightly degraded with delayed or missing channel state information. The performance degradation is larger when considering a basic bitstream extractor, which serves as reference ${ }^{1}$.

Index Terms-Cross-layer optimization, learning, QoE estimation,
\end{abstract} stochastic control, video streaming

\section{INTRODUCTION}

Efficient video streaming is an important application for next generation wireless networks [1]. Current mobile internet architectures were not designed to meet Quality of Experience (QoE) constraints on the received video, such as playout quality, limited delivery delay, or small zapping time. Efficient streaming over wireless networks is difficult due to the scarce radio resource and to the dynamic characteristics of the video contents and of the channel.

This paper proposes a cross-layer control mechanism to stream scalable videos to mobile receivers. Its goal is to maximize the quality of the received video while accounting for the variations of the characteristics of the transmitted content and of the channel. This control problem is addressed in the framework of Markov Decision Processes (MDPs) [2]. The optimal actions to apply to the system are learned via Reinforcement Learning (RL). Due to buffering delays, the quality of the decoded frames at receiver has to be inferred via an observation $(i)$ of the quality of the various scalability layers and (ii) of the level of queues at the Application (APL) and Medium Access Control (MAC) layers of the transmitter only.

The control consists in filtering the scalability layers of the encoded video. The considered model is more general than that considered in [3], since layers of one or several frames may be transmitted in each time slot. This allows to change the speed at which encoded frames are transmitted depending on the channel conditions and on the state of the buffers. To cope with the dynamic characteristics of the multimedia contents and of the wireless channel, as in [4], RL techniques are employed to dynamically update the optimal policy.

Encoded packet scheduling has been investigated in recent years to maximize the quality of the decoded multimedia streams given resource constraints. In [5], the problem of delay-sensitive rate control

${ }^{1}$ This work is supported by the European Commission under the MEDIEVAL project (grant agreement no FP7-258053). Michel Kieffer is partly supported by the Institut Universitaire de France. for transmission of real-time video over burst-error wireless channels is considered using constrained optimization of the encoding rate. The quantization parameters of the source coder are optimized dynamically to maximize the average quality while satisfying expected rate constraints using an accurate model of the rate-distortion (R-D) characteristics of the source.

In [6], a cross-layer scheduler for video transmission is presented. The time-varying characteristics of the channel are modeled by a Markov model, which state is fed back to the controller. Scalable video coders have been considered in [3], [7], focusing on the APL layer. A control policy is evaluated in the framework of MDPs. The level of the playback buffer at receiver side is exploited to help optimizing the performance of the system.

A QoE-based optimization framework for multi-user wireless video delivery is proposed in [8]. Transcoding and packet dropping are used in the rate adaptation scheme by investigating their impact on the perceived video quality in presence of constrained transmission resources. In [9], [10], and [11], focus is more on the MAC layer, since buffer management problems within the Radio Access Network (RAN) are considered. Video packets may be dropped depending on their priority and on the level of the MAC buffers.

In most of these papers, the channel and the receiver buffer states are used by the control process. Nevertheless, in real networks this information may reach the controller with a certain delay. When considering unicast transmission, various feedbacks may be obtained from the receiver. For example, RTCP [12] provides information on the level of buffers at APL layer. At MAC layer, HARQ ACK/NACK [13] may be used to infer the channel conditions. The associated delay may be of the order of tens to hundreds of milliseconds for HARQ ACK/NACK messages, and of one to several seconds for RTCP packets. Optimization of the video delivery in presence of delayed information may cause stability issues. MDP and learning in presence of delayed information with constant delay has been addressed in several paper, see, e.g., [14], [15]. Learning with constant reward delay is considered in [15]. A model-based RL algorithm is used in discrete- and continuous-valued state. Nevertheless, in the considered context, the scheduler is allowed to transmit more than one data unit (frames or GoPs) at each time slot leading to a time-varying delay between transmission and display.

Without channel state information, [16] shows that the observation of the level of the MAC buffer provides a satisfying estimate of the channel state. This prevents using delayed measurements. Moreover, the evaluation of the optimal layer filtering policy is performed offline. A way to tackle the problem of time-varying characteristics of the encoded videos and of the channel, is to learn and update on-line the optimal layer filtering policy. RL techniques are well-suited to update periodically the state-value function and the policy.

The remainder of the paper is organized as follows. Section II introduces the considered unicast video streaming. Section III recalls 
basic concepts linked to MDPs and RL. The model of the system is cast in the framework of MDPs in Section IV. Section V presents the performance of the proposed on-line layer filtering process and evaluates its robustness to variations of the characteristics of the wireless channel and of the transmitted content.

\section{SYSTEM DESCRIPTION}

Consider the video streaming system to mobile receivers sketched in Figure 1. The core network consists of a streaming server hosting a scalable video coder, a proxy, and a base station. Packets are transmitted through a wireless channel to a mobile client. Among the components of the base station (eNodeB [13]), we consider mainly the MAC buffer. The MAC scheduler of eNodeB, as well as its physical layer, its radio front-end, the wireless channel, the physical layer of the receiver, and the part of the MAC layer at receiver side managing ACK/NACK procedures are considered as belonging to the channel. Focusing on SNR scalability, our goal is to design a layer filtering algorithm to maximize the QoE of the decoded video at receiver side.

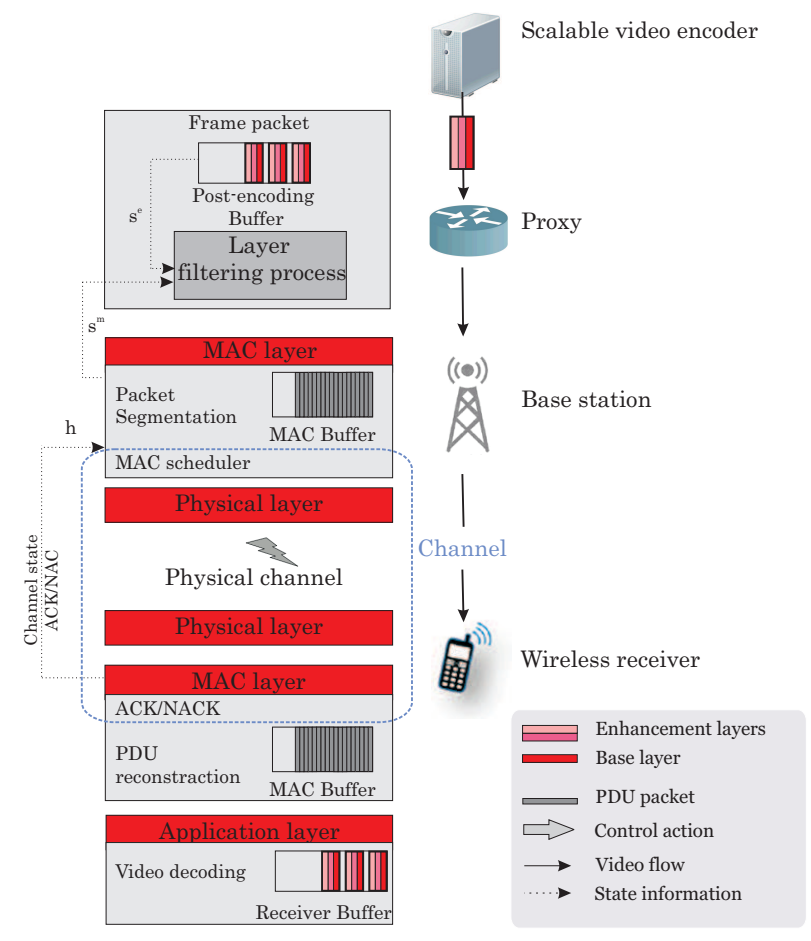

Figure 1. Scalable video transmission system to a mobile receiver

Streaming server: The video sequence is segmented into frames and encoded into $L$ layers: a base layer and $L-1$ enhancement layers. Frames are generated with a constant period of time $T$. The encoding parameters (quantization steps, frame rate, etc.) are controlled by the streaming server, independently of the remainder of the chain.

Proxy: The $L$ SNR layers are packetized and fed, via a lossless network, to the Post-Encoding (PE) buffer. The controller performs layer filtering within the proxy: for each frame, SNR layers may be sent, kept, or dropped. Layer filtering may also be performed in the base station (PDCP layer [13]). The layer filtering process should maximize the QoE at receiver side by taking into account most factors impacting it: frame type, number of SNR layers, lost packets due to PE and MAC buffer overflow, and effect of error and loss concealment.
Base station and channel: The base station contains a buffer dedicated to each user to perform rate and bandwidth allocation (MAC scheduling, see [17], [18]) among users. Packets transmitted by the layer filtering are fed from the PE buffer to the MAC buffer of the base station after being segmented into Packet Data Units (PDUs). One has to control the MAC buffer to avoid overflow in order to prevent PDUs from being dropped. PDUs are then transmitted to the mobile receiver via a wireless channel. When the channel state is used to control the layer filtering process, it has to be inferred, e.g., using some feedback from the mobile client or using the level of the MAC buffer at transmitter side.

Receiver: The mobile receiver stores correctly received PDUs in its own MAC buffer. Packet de-encapsulation and buffering in the buffer at APL layer is done as soon as all corresponding PDUs have been received. Complete or incomplete frames are then processed by the video decoder. Outdated packets are dropped, without being decoded. Some packet loss concealment may be put at work at the receiver side. Handover issues are not addressed: the streaming server is assumed to transmit video to a mobile receiver considered linked to the same base station during the whole streaming session.

\section{MDP AND LEARNING}

The video streaming system is modeled in the MDP framework. Time is slotted into discrete-time intervals of length $T$. The $t$-th time slot is the time interval $[t, t+1) T$. $T$ may be equal to the frame period, corresponding to the cadence of the encoder, or to the period at which the MAC scheduler delivers PDUs.

An MDP is a 4-tuple $(\mathcal{S}, \mathcal{A}, P, r)$, where $\mathcal{S}$ is the set of states of the considered system, $\mathcal{A}$ is the set of actions, $P\left(s_{t+1} \mid s_{t}, a_{t}\right)$ is the transition probability from $s_{t} \in \mathcal{S}$ at time $t$ to $s_{t+1} \in \mathcal{S}$ at time $t+1$, when the action $a_{t} \in \mathcal{A}$ is applied. Finally $r\left(s_{t}, a_{t}\right)$ is a reward function indicating the immediate reward obtained when applying $a_{t}$ in state $s_{t}$. Provided that all components of the MDP are clearly defined, the optimum policy may be evaluated, e.g., by value or policy iteration. Alternatively, when some components of the MDP are difficult to obtain, or are time-varying, a good policy may be obtained on-line by RL, see [2].

In the context of wireless video streaming, the characteristics of the video sequence and of the channel are time-varying. The policy that would be obtained via policy or value iteration for some transition probability matrix and some reward function under some source and channel conditions would probably not be well suited to other conditions. RL aims at estimating a good policy without requiring an accurate knowledge of the $P\left(s_{t+1} \mid s_{t}, a_{t}\right)$. There are several classes of on-line RL algorithms. This paper focuses on Temporal Difference (TD) learning [2], which aims at directly estimating the action-value function (or Q-function) $Q(s, a)$, indicating the expected long-term reward starting from $s$, taking the action $a$. The optimal policy is then derived by selecting the action maximizing $Q\left(s_{t}, a_{t}\right)$. Popular on-line algorithms in this category are SARSA and Q-learning [2].

With Q-learning, considered in what follows, the Q-function is updated at each time slot according to

$$
Q\left(s_{t}, a_{t}\right) \leftarrow Q\left(s_{t}, a_{t}\right)+\alpha_{t} \delta_{T D, t}
$$

with $\delta_{T D, t}=r_{t}\left(s_{t}, a_{t}\right)+\gamma \max _{a^{\prime} \in \mathcal{A}} Q_{t}\left(s_{t+1}, a_{t}^{\prime}\right)-Q\left(s_{t}, a_{t}\right)$, where $a_{t}^{\prime}$ is the greedy action in state $s_{t+1}$, which maximizes the current estimate of the Q-function; $\alpha \in[0,1]$ is a time-varying learning rate parameter and $\gamma$ is a discount factor indicating the relative importance of present and future rewards. $Q(s, a)$ can be initialized arbitrarily for all $(s, a) \in \mathcal{S} \times \mathcal{A}$. At the beginning of the learning process, the controller should go through the states many 
times in order to learn the optimal actions. During the exploration steps, the Q-learning rule in (1) is performed by executing actions in each state several times until $Q$ converges.

\section{MOdel OF THE STREAMING SySTEM}

The states of the system consists of the frame type $s^{\mathrm{f}}$, the level of the PE buffer $s^{\mathrm{e}}$, that of the MAC buffer $s^{\mathrm{m}}$, and the channel state $s^{\mathrm{h}}$. All state components are gathered in $\mathbf{s}=\left(s^{\mathrm{f}}, s^{\mathrm{e}}, s^{\mathrm{m}}, s^{\mathrm{h}}\right) \in \mathcal{S}$. The actions indicate the number of scalability layers to transmit, keep or drop from the PE buffer.

\section{A. States}

The frame type $s^{\mathrm{f}}$ (I, P, or B) is useful to describe the impact of a frame losses on the video quality. In a GoP, transitions between frame types may be described by a stationary Markov process [19]. In what follows, the structure of the GOP is assumed constant for the whole video sequence.

The state of the PE buffer is $s^{\mathrm{e}} \in \mathcal{S}^{\mathrm{e}}$ and that of the MAC buffer is $s^{\mathrm{m}} \in \mathcal{S}^{\mathrm{m}}$. Here, $s^{\mathrm{e}}$ describes the number of encoded frames stored; this helps controlling the delay introduced within the system. The state of the MAC buffer indicates the number of PDUs or of bits (PDUs are assumed to have the same size) in the buffer.

The channel state $s_{t}^{\mathrm{h}}$ describes the time-varying channel conditions, such as the rate, probability of error, capacity, etc., assumed constant in $[t, t+1)$. Here, $s_{t}^{\mathrm{h}}$ corresponds to the channel rate modeled as the realization of an $N_{\mathrm{h}}$-state Markov chain as in [20]. At time $t$, the state $s_{t}^{\mathrm{h}} \in \mathcal{H}=\left\{1, \ldots, N_{\mathrm{h}}\right\}$ represents a rate within the set $\mathcal{R}^{c}=\left\{R_{0}^{\mathrm{c}}, \ldots, R_{N_{h}}^{c}\right\}$. The transition probability $p_{k, \ell}=p\left(s_{t}^{\mathrm{h}}=\right.$ $\left.\ell \mid s_{t-1}^{\mathrm{h}}=k\right)$ from state $\ell \in\left\{1, \ldots, N_{h}\right\}$ to state $k \in\left\{1, \ldots, N_{h}\right\}$ has usually to be estimated on-line.

Three scenarii concerning the knowledge of the state of the channel are considered: (i) instantaneously available, $s_{t}^{\mathrm{h}}$ is available at time $t$, which requires feedback with very short delay; (ii) delayed, only $s_{t-\delta}^{\mathrm{h}}$ is available at time $t$, with $\delta>0$ some feedback delay, which is more realistic; (iii) unknown, no channel state feedback is available.

\section{B. Actions}

In the proposed model, several frames may be transmitted in each time slot, allowing to speed up or slow down the frame scheduling according to the channel and buffer conditions. The layer filtering process has to determine the number of layers among the $F$ oldest frames stored in the PE buffer to send to the MAC buffer.

The vector of actions $a_{t}=\left(a_{\ell, f}\right)_{t} \in \mathcal{A}^{L \times F}$ with $\ell \in\{1, \ldots, L\}$ and $f \in\{1, \ldots, F\}$ taken in $[t, t+1)$ represents the filtering decisions. For the $\ell$-th SNR layer and the index $f$ of the frame in the PE buffer ( $f=1$ is the oldest one and $f=F$ the earliest), $a_{\ell, f}=1$ indicates a transmitted layer, $a_{\ell, f}=-1$ a dropped layer, and $a_{\ell, f}=0$ indicates that the layer is temporarily kept in the buffer.

A layer may be decoded only if the corresponding higherimportance layers have already been decoded. When some layer is dropped from the PE buffer, the actions are designed in such a way that all refinement layers of the dropped layer belonging to the same frame are also dropped.

\section{Estimation of the reward}

$\mathrm{RL}$ requires for each time slot some reward $r_{t}\left(s_{t}, a_{t}\right)$ provided by the system to update $Q\left(s_{t}, a_{t}\right)$. Ideally, $r_{t}\left(s_{t}, a_{t}\right)$ should be $(i)$ related to the user QoE (level of satisfaction) after applying action $a_{t}$ when the state is $s_{t}$ and (ii) fed back instantaneously by the receiver. QoE information may be obtained considering the PSNR, the SSIM, or other metrics [21], [22], [23]. Automatic QoE measurement tools [24] may be particularly useful in this context. Unfortunately, even with such tools, due to buffering, the action $a_{t}$ will have an impact on the user QoE only after some delay $\delta_{t}$. This reward evaluation delay is time-varying, since the PE buffer is allowed to temporarily keep frames or to transmit several frames in the same time slot. Let $r_{t}^{\delta_{t}}\left(s_{t}, a_{t}\right)$ be the reward provided by the receiver after a delay $\delta_{t}$ when the state at time $t$ is $s_{t}$ and the action $a_{t}$.

To address the problem of delayed rewards, the QoE (and the reward) $r_{t}^{\delta_{t}}\left(s_{t}, a_{t}\right)$ obtained by the receiver at time $t+\delta_{t}$ is predicted at time $t$ at transmitter side $\widehat{r}_{t}\left(s_{t}, a_{t}\right)$. To facilitate prediction, we assume that the QoE can be evaluated frame by frame (which is not true for a video, since the motion plays an important role). We assume further that an overflowed MAC buffer drops all entering frames and that an overflowed PE buffer drops its oldest frame (the drop action is forced for that frame). Finally, retransmissions and adaptive modulation and coding schemes are used at MAC layer to ensure the delivery of all PDUs from the MAC buffer to the receiver.

Several cases have now to be considered.

1) Transmission/drop of a single frame: Consider that layers from a single frame are transmitted from the post-encoding buffer to the MAC buffer. In absence of overflow or empty buffers, the reward evaluation delay remains constant $\delta_{t}=\delta_{t-1}$ and the QoE evaluated at the encoder is equal that evaluated at the decoder

$$
\widehat{r}_{t}\left(s_{t}, a_{t}\right)=r_{t}^{\delta_{t}}\left(s_{t}, a_{t}\right)=\sum_{\ell=1}^{L} \max \left(0, a_{\ell, 1, t}\right) q_{t}\left(s_{t}^{\mathrm{f}}, \ell\right)
$$

where $q\left(s^{\mathrm{f}}, \ell\right)$ is the additional QoE measure provided by the transmission of layer $\ell$ from a frame of type $s^{\mathrm{f}}$.

Assume now that a single frame is still transmitted and that layers are dropped either by the layer filtering process, or due to buffer overflow. When the base layer remains, (2) is still valid. When it is dropped, concealment is performed at receiver side, and $\delta_{t}=\delta_{t-1}$. Several concealment techniques may be used. Here frame copy [25] is considered and the QoE of the current frame is assumed to be equal to that of the previous frame reduced by a factor $\lambda\left(s^{\mathrm{f}}\right)$ depending on the type of the lost frame (lost I frames will have more impact on the next frames than lost $\mathrm{P}$ frames). One then gets

$$
\widehat{r}_{t}\left(s_{t}, a_{t}\right)=\widehat{r}_{t-1}\left(s_{t-1}, a_{t-1}\right)-\lambda\left(s_{t}^{\mathrm{f}}\right) .
$$

2) Temporarily kept frames: When frames are neither transmitted to the MAC buffer, nor dropped (intentionally or as a consequence of post-encoder buffer overflow), the reward evaluation delay decreases $\delta_{t}=\delta_{t-1}-1$ for the next frame transmitted from the PE buffer to the MAC buffer. As a consequence, estimating $r_{t}^{\delta_{t}}\left(s_{t}, a_{t}\right)$ is quite difficult, since no frame is transmitted at time $t$. The impact of the QoE at the receiver will be via the next transmitted frames, for which no decision has been considered at time $t$. Thus, we consider that the reward is the average $\mathrm{QoE}$ of the next frame, i.e.,

$$
\widehat{r}_{t}\left(s_{t}, a_{t}\right)=\frac{\widehat{r}_{t-1}\left(s_{t-1}, a_{t-1}\right)-\lambda\left(s_{t}^{\mathrm{I}}\right)+\sum_{L^{\prime}=1}^{L} \sum_{\ell=1}^{L^{\prime}} q_{t}\left(s_{t}^{\mathrm{I}}, \ell\right)}{L+1} .
$$

The first term in (4) corresponds to a dropped next frame and is equal to (3), the second term corresponds to a number of layers transmitted going from 1 to $L$ with rewards as in (2).

3) Transmission of several frames: When layers of several frames are transmitted from the PE buffer to the MAC buffer during the same time slot, the reward evaluation delay increases $\delta_{t}=\delta_{t-1}+1$ for the next frame transmitted, since more frames are put in the MAC buffer. This decision will impact the QoE of several frames at receiver side. It is again quite difficult to evaluate precisely $r_{t}^{\delta_{t}}\left(s_{t}, a_{t}\right)$. The transmission of layers of several frames should not lead to jitter in the 


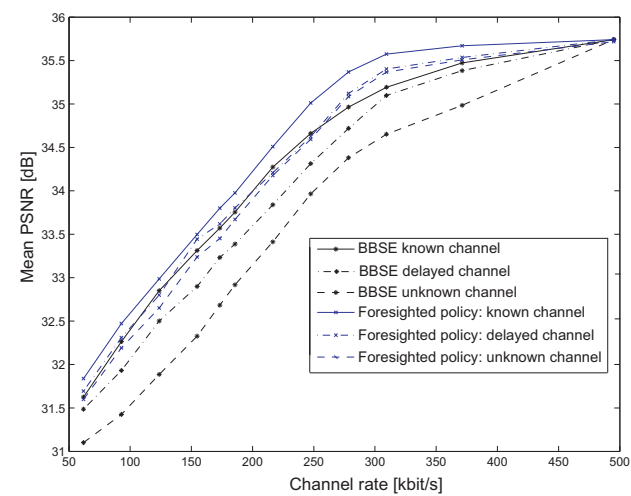

Figure 2. PSNR of the decoded Foreman sequence, control policy obtained by RL and that of the BBSE, considering 3 channel scenarii.

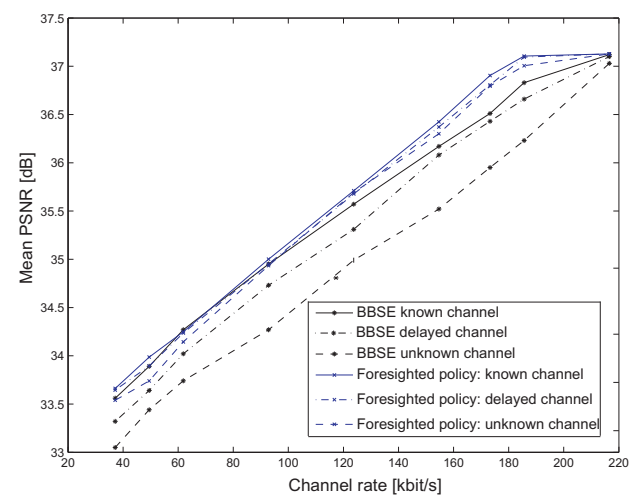

Figure 3. PSNR of the decoded Mother\&Daughter sequence, control policy obtained by RL and that of the BBSE, considering 3 channel scenarii.

quality of the decoded frames. Thus, the estimated reward is taken as the minimum among the rewards obtained for each individual frames

$$
\widehat{r}_{t}\left(s_{t}, a_{t}\right)=\min _{f \in\{1, \ldots F\}}\left(\sum_{\ell=1}^{L} \max \left(0, a_{\ell, f, t}\right) q_{t+f-1}\left(s_{t}^{\mathrm{I}}, \ell\right)\right) .
$$

This prevents sending during a time slot all layers from the first frame and only a single layer from the next frames.

\section{EXPERIMENTAL RESULTS}

The performance of the proposed layer filtering process has been evaluated on the Foreman.qcif and Mother \& Daughter.qcif sequences at 30 fps using the H.264/SVC encoder (JSVM 9.19) [26] with three MGS scalability layers per frame $(L=3)$. The period at which the controller is operating is $T=1 / 30 \mathrm{~s}$. IPPP GoPs of 16 frames are considered. To avoid the drift due to SNR layer filtering, all frames are encoded as key pictures for which motion compensation is performed using only the base layer of the previous frames.

\section{A. Reference Basic BitStream Extractor (BBSE)}

The BBSE provided in the JSVM [26] serves as reference. It extracts SVC layers according to a specific priority and accounting for the level of the MAC buffer. The priorization is done according to high-level syntax elements: dependency, temporal, and quality ids. Here, NAL units are ordered based on their quality level and are stored in the MAC buffer provided that it does not overflow.

\section{B. Simulation conditions}

The channel is described by a two-state Markov model, with a bad (B) state with channel rate $R_{\mathrm{B}}^{\mathrm{c}}$ and $\operatorname{good}(\mathrm{G})$ state with

\begin{tabular}{|l|l|}
\hline$a^{(1)}=(0,0,0,0,0,0)$ & $a^{(7)}=(1,1,1,1,1,1)$ \\
\hline$a^{(2)}=(1,-1,-1,0,0,0)$ & $a^{(8)}=(1,-1,-1,1,-1,-1)$ \\
\hline$a^{(3)}=(1,1,-1,0,0,0)$ & $a^{(9)}=(1,1,-1,1,-1,-1)$ \\
\hline$a^{(4)}=(1,1,1,0,0,0)$ & $a^{(10)}=(1,1,-1,1,1,-1)$ \\
\hline$a^{(5)}=(1,1,1,1,-1,-1)$ & $a^{(11)}=(-1,-1,-1,0,0,0)$ \\
\hline$a^{(6)}=(1,1,1,1,1,-1)$ & \\
\hline
\end{tabular}

CONSIDERED ACTIONS WHEN $L=3$ AND $F=2$.

channel rate $R_{\mathrm{G}}^{\mathrm{c}}$. The state transitions occur with a period $T$ and with probabilities $P(G \mid G)=0.9$ and $P(B \mid B)=0.8$, leading to the stationary probabilities $P(G)=0.66$ and $P(B)=0.33$. As indicated in Section IV-A, the state of the channel may be instantaneously available, delayed, or unknown, depending on the considered scenario.

The PE and MAC buffers contain at most $B^{\mathrm{e}}=25$ frames and $B^{\mathrm{m}}=500$ PDUs respectively. The PDUs are assumed to be static with size 336 bits, which is consistent with the 3GPP radio link control protocol specification [27]. To get a model with reduced state space and accelerate the convergence of RL, the state of the PE buffer is quantized to two intervals $\left[0,22\left[\right.\right.$ represented by $s^{\mathrm{e}}=1$, indicating a satisfying occupancy and $[22,25]$, represented by $s^{\mathrm{e}}=2$, indicating a buffer close to overflow. As the MAC state transitions depend on the encoded frame size contrary to the PE buffer state, a finer quantization is considered for the state of the MAC buffer. Moreover, when the channel state is unknown, the control has to rely on the observation of the state of the MAC buffer only. MAC buffer states are quantized into five intervals. The fifth is smaller than the others to anticipate overflow and prevent PDUs from being dropped.

The number of possible actions is kept small to limit the learning complexity. The layers of at most two frames in the PE buffer may be fed to the MAC buffer, i.e., $F=2$. The action for each layer is organized as $a_{t}=\left(a_{1,1, t}, \ldots, a_{3,1, t}, a_{1,2, t}, \ldots, a_{3,2, t}\right) \in \mathcal{A}$ with $a_{\ell, f, t}=\{-1,0,1\}$. When only the base layer of a frame is transmitted, the other layers are dropped, see Table V-B. For example $a^{(9)}$ indicates the transmission of two layers of the oldest frame and of the highest priority layer of the next frame.

The reward involves the PSNR of the two last frames, but any other video quality metric may be used in the proposed learning process. The value of the PSNR reduction $\lambda\left(s_{t}^{\mathrm{I}}\right)$ depends on the frame type. Off-line measurements are performed using different sequences with frame copy concealment leading to $\lambda(\mathrm{I})=15 \mathrm{~dB}$ when an I frame is lost and $\lambda(\mathrm{P})=8 \mathrm{~dB}$ when a $\mathrm{P}$ frame is lost.

\section{Results}

On-line Q-learning is performed over 5000 time slots (on Foreman and Mother\&Daughter sequences by repeating the sequences from the beginning after 300 frames). $Q(s, a)$ is initialized to zero for each state-action pair. The discount factor is set to $\gamma=0.9$.

Considering the three level of knowledge of the channel state, Figures 2 and 3 compare the performance of the streaming server using the policy obtained by RL and that of the BBSE. Different channel rates are considered form 50 to $500 \mathrm{kbit} / \mathrm{s}$ for Foreman and from 40 to $250 \mathrm{kbit} / \mathrm{s}$ for Mother\&Daughter. In Figures 2 and 3, a separate learning is performed for each value of the rate.

As shown in Figures 2 and 3 and in Table II when the channel state is instantaneously known, the proposed RL-based scheme outperforms the BBSE in most cases for both video sequences. The gain of the proposed scheme compared to the BBSE is mainly due to more accurate SNR layer selection, which better accounts for the 


\begin{tabular}{|c|c|c|c|c|}
\hline Channel state & \multicolumn{2}{|c|}{ Foreman } & \multicolumn{2}{c|}{ Mother \& Daughter } \\
\hline & PSNR & Rate & PSNR & Rate \\
\hline Known & 0.4 & 60 & 0.39 & 22 \\
\hline Delayed & 0.54 & 70 & 0.43 & 33 \\
\hline Unknown & 0.91 & 120 & 0.84 & 42 \\
\hline
\end{tabular}

Table II

AVERAGE GAIN IN PSNR (DB) AND RATE (KBIT/S) OF THE POLICY OBTAINED BY RL COMPARED TO THE BBSE.

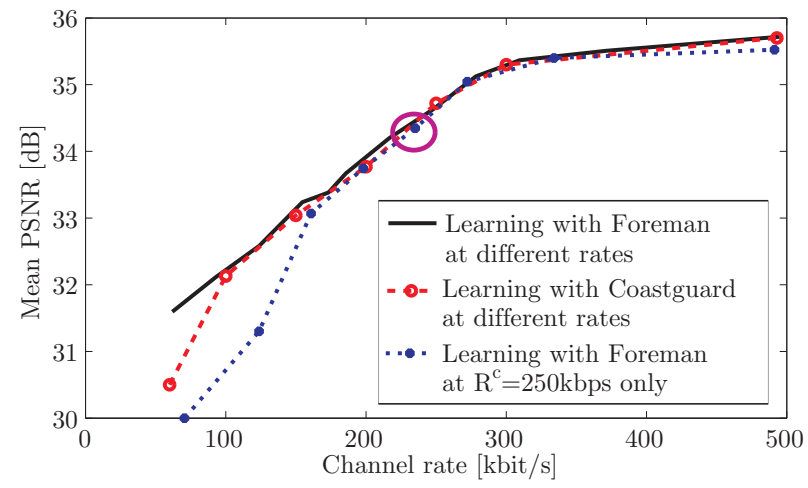

Figure 4. PSNR as a function of channel rate for Foreman sequence when optimal policy is learned with Foreman at each channel rate (plain), with Coastguard at each channel rate (dashed red) and with Coastguard at $R^{c}=$ $250 \mathrm{kbps}$ only (dotted blue).

contribution of each layer to the video quality, and for the foresighted policy obtained by RL.

To evaluate the robustness of the proposed approach to variations of the characteristics of the system, the policy obtained by learning with a video sequence, here coastguard.qcif is used, and applied to Foreman when the channel state is unknown, as illustrated by the red dashed curve in Figure 4. The behavior in presence of rate variations is similar to that observed using only Foreman for learning, showing the robustness to variations of the transmitted content.

To evaluate the robustness to variations of the channel rate, the policy learned for $R^{c}=250 \mathrm{kbps}$ is applied for different channel rates when the channel state is unknown for the Foreman sequence, see Figure 4. The results obtained when the policy is learned at each channel rate and when it is learned using only $R^{c}=250 \mathrm{kbps}$ are very close, except when the channel rate becomes very different from that used for the learning process. The RL based control process is thus quite robust to moderate variations of the channel characteristics.

\section{CONCLUSION}

A RL solution for scalable video transmission over a time-varying wireless channel is proposed. Experiments show that with delayed or without channel state information, the performance obtained with the policy obtained by RL is only slightly degraded compared to a case where the channel state information is available. The performance degradation is larger using a BBSE.

The robustness to variations of the characteristics of the channel and of the video sequence has been shown experimentally. The QoE metric considered in this paper is the PSNR, but other quality metrics could readily be used in the learning process, provided that they allow the QoE of the receiver to be predicted at the transmitter. Implementation of the proposed layer filtering process in a prototype LTE network including a functional eNodeB and UE is planed.

\section{ACKNOWLEDGMENTS}

The authors would like to thank Prof. M. van der Schaar for valuable discussions on dynamic programming.

\section{REFERENCES}

[1] N. Amram, B. Fu, G. Kunzmann, T. Melia, D. Munaretto, S. Randriamasy, B. Sayadi, J. Widmer, and M. Zorzi, "QoE-based transport optimization for video delivery over next generation cellular networks," in IEEE SCC, Kerkyra, 2011, pp. $19-24$.

[2] R. Sutton and A. Barto, Reinforcement Learning: An Introduction. MIT Press, 1998.

[3] P. de Cuetos and K. Ross, "Optimal streaming of layered video: joint scheduling and error concealment," in ACM MM, Berkeley, 2003, pp. 2 $-8$.

[4] F. Fu and M. Van der Schaar, "Structural solutions to dynamic scheduling for multimedia transmission in unknown wireless environments," Multimedia Systems and Applications Papers in Computer and Information Science, vol. abs/1008.4406, 2010.

[5] H. Chi-Yuan, A. Ortega, and M. Khansari, "Rate control for robust video transmission over burst-error wireless channels," IEEE JSAC, vol. 17 no. 5, pp. $756-773,1999$.

[6] W. Kumwilaisak, Y. Hou, Q. Zhang, W. Zhu, C.-C. Kuo, and Y.-Q. Zhang, "A cross-layer quality-of-service mapping architecture for video delivery in wireless networks," IEEE JSAC, vol. 21 , no. 10 , pp. $1685-$ 1698, December 2003.

[7] N. Changuel, C. Mastronarde, M. van der Shaar, B. sayadi, and M. kieffer, "End-to-end stochastic scheduling of scalable video over timevarying channels," in ACM MM, Florence, 2010, pp. 731 - 734.

[8] S. Thakolsri and W. Kellerer, "QoE-based rate adaptation scheme selection for resource-constrained wireless video transmission," in ACM $M M$, Florence, 2010, pp. $783-786$.

[9] G. Liebl, H. Jenkac, T. Stockhammer, and C. Buchner, "Radio link buffer management and scheduling for wireless video streaming," in $P V$ Workshop, Irvine, 2004, pp. 255 - 277.

[10] Z. Orlov and M. C. Necker, "Enhancement of video streaming QoS with active buffer management in wireless environments," in Proc EW, Paris, 2007.

[11] S. Yerima and K. Al-Begain, "Dynamic buffer management for multimedia qos in beyond $3 \mathrm{G}$ wireless networks," IJCS, vol. 36, no. 4, pp. 378 - 387, November 2009.

[12] H. Schulzrinne and S. Casner, RTP profile for audo and video conferences with minimal control, IETF, July 2003, rFC 3551.

[13] S. Sesia, I. Toufik, and M. Baker, LTE, The UMTS Long Term Evolution : From theory to practice. Wiley, February 2009.

[14] E. Altman and P. Nain, "Closed-loop control with delayed information," in ACM SIGMETRICS, New York, June 1992, pp. 193 - 204.

[15] T. J. Walsh, A. Nouri, L. Li, and L. M. L., "Learning and planning in environments with delayed feedback," AAMAS, vol. 18, no. 1, pp. $83-$ 105, August 2009.

[16] N. Changuel, N. Mastronarde, M. van der Schaar, B. Sayadi, and M. Kieffer, "Adaptive scalable layer filtering process for video scheduling over wireless networks based on MAC buffer management," in IEEE ICASSP, Prague, 2011, pp. $2352-2355$.

[17] H. Fattah and C. Leung, "An overview of scheduling algorithms in wireless multimedia networks," IEEE Wireless Communications, vol. 9, no. 5 , pp. $76-83$, October 2002.

[18] W. Ajib and D. Haccoun, "An overview of scheduling algorithms in MIMO-based fourth-generation wireless systems," IEEE Network, vol. 19 , no. 5 , pp. $43-48$, September 2005.

[19] D. Turaga and T. Chen, "Hierarchical modeling of variable bit rate video sources," in PV Workshop, Kyongju, 2001.

[20] J. Cabrera, A. Ortega, and J. Ronda, "Stochastic rate-control of video coders for wireless channels," IEEE trans. on circuits and systems for video technology, vol. 12, no. 6, pp. 496 - 510, June 2002.

[21] ITU-T, "Objective perceptual multimedia video quality measurement in the presence of a full reference," ITU-T Rec. J.247 (08/08), Tech. Rep. Rec. J.247, 2008.

[22] G. J. Sullivan and T. Wiegand, "Rate-distortion optimization for video compression," IEEE Signal Processing Magazine, vol. 15, no. 6, pp. 74 - 90, November 1998.

[23] G. Winkler, Image Analysis, Random Fields and Dynamic Monte Carlo Methods: A Mathematical Introduction, 2nd ed., S. Verlag, Ed. Springer, 2005, vol. 27 of Applications of Mathematics.

[24] K. Singh, A. Ksentini, and B. Marienval, "Quality of experience measurement tool for svc video coding," in IEEE ICC, 2011, pp. 1 - 5.

[25] Z. Wu and J. M. Boyce, "An error concealment scheme for entire frame losses based on H.264/AVC," in IEEE ISCS, Kos, 2006, pp. 1 - 4.

[26] J. Vieron, M. Wien, and H. Schwarz, JSVM9 software, Joint Video Team (JVT) of ISO/IEC MPEG \& ITU-T VCEG,, JVT-V203, January 2007.

[27] "Radio link control (RLC) protocol specification (release 5)," 3GPP TS 25.322 V5.12.0, Tech. Rep., 2005. 